\title{
Huertos universitarios y necesidades humanas: una aproximación bibliográfica y vivencial desde el huerto agroecológico de la Universidad Veracruzana en México
}

\author{
Juan Camilo Fontalvo-Buelvas \\ huertobiologia@uv.mx \\ Universidad Veracruzana (Veracruz, México) \\ (iD https://orcid.org/0000-0002-9818-0489 \\ Yadeneyro de la Cruz Elizondo \\ ydelacruz@uv.mx \\ Universidad Veracruzana (Veracruz, México) \\ (iD) https://orcid.org/0000-0002-2034-6637
}

\begin{abstract}
Resumen
Los huertos universitarios son espacios de pequeña extensión, que han sido conformados en primera instancia con fines educativos y productivos; sin embargo, estas áreas verdes citadinas ofrecen mucho más que aprendizajes y alimentos. Desde la perspectiva del desarrollo humano, estos espacios cultivados podrían ser un canal para satisfacer de forma simultánea necesidades humanas fundamentales. Por tanto, el objetivo de este estudio es explicar la relación entre los huertos universitarios y la satisfacción de necesidades humanas fundamentales a través de una aproximación bibliográfica y vivencial desde el Huerto Agroecológico de la Facultad de Biología de la Universidad Veracruzana en México. Esta investigación exploratoria se realizó siguiendo la metodología de estudio de caso, la cual distingue cuatro etapas: formulación de la pregunta de reflexión, unidad de análisis, métodos e instrumentos de recolección de la información y análisis de la información. Los resultados mostraron que el huerto universitario puede responder primordialmente a la satisfacción de las necesidades de ocio, entendimiento, participación e identidad, y, en menor medida, a las necesidades de subsistencia, protección, creación, libertad y afecto. Algunos beneficios asociados a la satisfacción de necesidades en estos espacios cultivados están relacionados con la sustentabilidad urbana y el bienestar social, aspectos fundamentales que promueve la agroecología urbana. Las conjeturas aquí descritas pueden representar un punto de partida importante para una discusión más profunda sobre la relación existente entre los huertos y las necesidades humanas. En un siguiente momento será necesario visualizar aquellas herramientas metodológicas que permitan describir los procesos y fenómenos sociales aquí suscitados.
\end{abstract}

\section{Palabras clave}

Universidad, huerta, bienestar, sustentabilidad, agroecología urbana.

\section{(c) (i)}




\title{
University vegetable patches and human needs: a bibliographic and experiential approach from the agroecological garden at Universidad Veracruzana in Mexico
}

\author{
Juan Camilo Fontalvo Buelvas \\ huertobiologia@uv.mx \\ Universidad Veracruzana (Veracruz, México) \\ (iD https://orcid.org/0000-0002-9818-0489 \\ Yadeneyro de la Cruz Elizondo \\ ydelacruz@uv.mx \\ Universidad Veracruzana (Veracruz, México) \\ (iD https://orcid.org/0000-0002-2034-6637
}

\begin{abstract}
University vegetable patches are small spaces which have been created firstly for educational and productive purposes. However, these city green areas offer much more than learning and food. From a human development perspective, these cultivated spaces could be a channel to simultaneously satisfy fundamental human needs. Therefore, the objective of this study is to explain the relationship between university gardens and the satisfaction of fundamental human needs through a bibliographic and experiential approach from the Agroecological Garden of the Faculty of Biology at Universidad Veracruzana in Mexico. This exploratory research was carried out following the case study methodology, which distinguishes four stages: formulation of the reflection question, unit of analysis, information gathering methods and instruments, and information analysis. The results showed that vegetable patches at universities can respond primarily to the satisfaction of leisure, understanding, participation and identity needs, and, to a lesser extent, to the needs of subsistence, protection, creation, freedom, and affection. Some benefits associated with meeting needs in these cultivated spaces are related to urban sustainability and social well-being, fundamental aspects promoted by urban agroecology. The conjectures described here may represent an important starting point for a more in-depth discussion of the relationship between gardens and human needs. In the future it will be necessary to visualize those methodological tools that allow describing the processes and social phenomena raised here.
\end{abstract}

\section{Palabras clave}

University, vegetable patch, well-being, sustainability, urban agroecology. 


\section{Introducción}

En los últimos años, se ha evidenciado un auge sorprendente de huertos en diferentes instituciones educativas de Iberoamérica, al punto de convertirse en una herramienta didáctica importante para docentes dentro de sus quehaceres pedagógicos (Aldea, 2012). Los huertos han permeado todos los niveles educativos, y se encuentran en preescolares (Hilerio-Cruz, 2017), primarias (Vílchez y Escobar, 2014), secundarias (Reina et al., 2017) e incluso universidades (Eugenio-Gozalbo y Aragón-Núñez, 2016). Esto pone de manifiesto que el huerto, como espacio de enseñanza y aprendizaje (Lafuente-Miranda, 2017), es útil y práctico para atender necesidades educativas de nińos, adolescentes, jóvenes y adultos. Por tanto, el huerto resulta ser versátil, ya que tiene la capacidad de adaptarse con rapidez y facilidad a distintas funciones, en este caso, a distintos escenarios y necesidades de aprendizaje, una propiedad muy valorada y apreciada por los docentes (López et al., 2011).

Estos espacios de cultivo en el contexto universitario han demostrado ser eficientes para promover aspectos preponderantes como la participación, el diálogo de saberes, los aprendizajes significativos, el ambiente pedagógico, la interdisciplinariedad y la transversalidad de ejes temáticos como la sustentabilidad (Merçon et al., 2012). Asimismo, el huerto es idóneo para desarrollar en los estudiantes competencias teóricas y heurísticas, así como importantes competencias axiológicas relacionadas con la cooperación, el trabajo en equipo, el respeto, la justicia, la democracia, e incluso el sentido estético y la cultura ambiental (Botella et al., 2007; Barrón y Muñoz, 2015). Estos beneficios educativos son posibles cuando el docente, de forma planificada, logra articular el huerto con el plan de estudios, sus asignaturas y temas concretos. Dicha articulación puede conseguirse mediante sesiones de docencia, prácticas escolares, proyectos de investigación o actividades de extensión a la sociedad civil, en las que el huerto es el eje del aprendizaje (Fontalvo-Buelvas, 2021).

Los huertos son también pequeñas áreas verdes que ofrecen servicios ecosistémicos importantes (Camps-Calvet et al., 2016), ya que promueven la biodiversidad (Montagnini y Metzel, 2015), la regeneración urbana (Álvarez-Ferri, 2012), la calidad ambiental (Malca, 2013) y el cierre de ciclos del metabolismo urbano (Deelstra, 1987). Por otro lado, desde la perspectiva del desarrollo humano se ha mencionado que estos espacios pueden atender necesidades humanas fundamentales (Morán, 2014). En este sentido, los huertos pueden ser uno de esos canales mediante los cuales se contribuye a la satisfacción de las necesidades de subsistencia (Martínez y Juan, 2005), ocio (Richter y Cuenca, 2018), identidad (Moctezuma-Pérez, 2010) y conocimiento principalmente (Merçon et al., 2012). Ahora bien, es necesario resaltar que la capacidad de los huertos para proveer alimentos, salud, seguridad y estabilidad económica dependerá del número de personas asociadas, el tamaño del huerto, los tipos de cultivos y las estrategias de repartición o comercialización.

Maslow (1943) fue el primero en expresar que existe una jerarquía de necesidades humanas. Él las representa a través de la conocida Pirámide de Maslow, que va desde necesidades básicas (fisiológicas) a superiores (seguridad, afiliación, reconocimiento y 
autorrealización). Por su parte, Sen (1987) acuñó el término capacidades básicas y, aunque no describió un conjunto de capacidades específicas, a partir de sus obras se han recopilado algunos ejemplos: "Comer bien y no padecer enfermedades evitables", "participar en la vida de la comunidad", "respetarse a uno mismo", "capacidad para llevar una vida responsable", el empleo, la libertad de elegir y "capacidad de lectura y escritura" (Groppa, 2005). Desde otra perspectiva, Doyal y Gough (1991) describen que las necesidades básicas se realizan mediadas por las necesidades intermedias, las cuales son agua limpia y comida nutritiva, vivienda protectora, ambiente laboral no riesgoso, medio ambiente no riesgoso, cuidado adecuado de la salud, seguridad en la infancia, relaciones primarias significativas, seguridad física, seguridad económica, educación apropiada, parto seguro y cuidado neonatal. Por otra parte, Nussbaum (1998) señala un grupo de capacidades básicas con las que debería contar todo ser humano, no como una medición del bienestar sino como un objetivo político. Tales capacidades son vida, salud corporal, integridad corporal, sentidos, imaginación, sentimientos, emociones, razón práctica, afiliación, otras especies, juego y control sobre el propio entorno.

Desde una perspectiva ontológica, Max-Neef (1992) simplificó nueve necesidades humanas fundamentales e interdependientes en las que ninguna se sobrepone a otra, ninguna es más importante y todas son universales. Estas son subsistencia, protección, afecto, entendimiento, participación, ocio, creación, identidad y libertad; tales necesidades no han variado históricamente, mas lo que sí puede variar son los canales para satisfacer o inhibir tales necesidades. En este sentido, Max-Neef (1993) señala distintos tipos de satisfactores, tales como los singulares, aquellos que estimulan la satisfacción de una necesidad en particular siendo neutros en relación con la satisfacción de otras; los sinérgicos, para referirse a aquellos que, por la manera en que satisfacen una necesidad en particular, ejercitan y favorecen la satisfacción paralela de otras necesidades; los pseudosatisfactores, medios que generan una falsa sensación de satisfacción de una necesidad determinada; los inhibidores, canales que, por el modo en que satisfacen una necesidad determinada, dificultan seriamente la posibilidad de satisfacer otras necesidades; y los violadores, aquellos que, por su configuración o efectos colaterales, no solo impiden la satisfacción de la necesidad para la que fueron diseñados, sino que imposibilitan la satisfacción de otras necesidades.

Ahora bien, ¿̇de qué manera estas necesidades y/o capacidades se vinculan con los huertos universitarios?, ¿qué beneficios se derivan en el contexto universitario cuando se logran desarrollar estas capacidades en los huertos?, ¿qué condiciones deben favorecerse para que estos espacios puedan atender tales necesidades? Estas son algunas preguntas que intentaremos resolver en los siguientes apartados. Así, el objetivo principal de este discurso es explicar la relación entre los huertos universitarios y la satisfacción de necesidades humanas fundamentales a través de una aproximación bibliográfica y vivencial desde el Huerto Agroecológico de la Facultad de Biología de la Universidad Veracruzana en México. 


\section{Metodología}

\section{1. Área de estudio}

El Huerto Agroecológico es un aula-laboratorio que, desde 2014, tiene como objetivo apoyar las funciones sustantivas de la Facultad de Biología en la región Xalapa de la Universidad Veracruzana en México (Figura 1). Se trata de un espacio dedicado a la agricultura urbana, con una extensión de $303 \mathrm{~m}^{2}$, que se encuentra dividido en distintas áreas, como banco de semillas, almácigos, compostas, captación de agua de lluvia, educativa y productiva (camas de cultivo). Este agroecosistema tiene vegetación nativa, árboles frutales, hortalizas, arvenses, plantas alimenticias no convencionales, así como medicinales, aromáticas y ornamentales (Fontalvo-Buelvas, 2021). Su diseño y manejo es agroecológico, por lo que se implementan una serie de buenas prácticas como el uso de semillas nativas, abonado orgánico, acolchado natural del suelo, labranza reducida, riego inteligente, rotación y asociación de cultivos, barreras y cercas vivas, manejo agroecológico de plagas y enfermedades, así como comercio próximo, justo y solidario. Este espacio cultivado cuenta con un colectivo asociado integrado por un docente y una plantilla de alrededor de 20 estudiantes que se va renovando semestralmente. El colectivo de este huerto se encuentra articulado y colabora de forma permanente con la Red de Huertos de la Universidad Veracruzana, la red de huertos escolares y comunitarios de Xalapa, la Red de Agricultura Urbana y Periurbana de Xalapa, y la Red Internacional de Huertos Escolares.

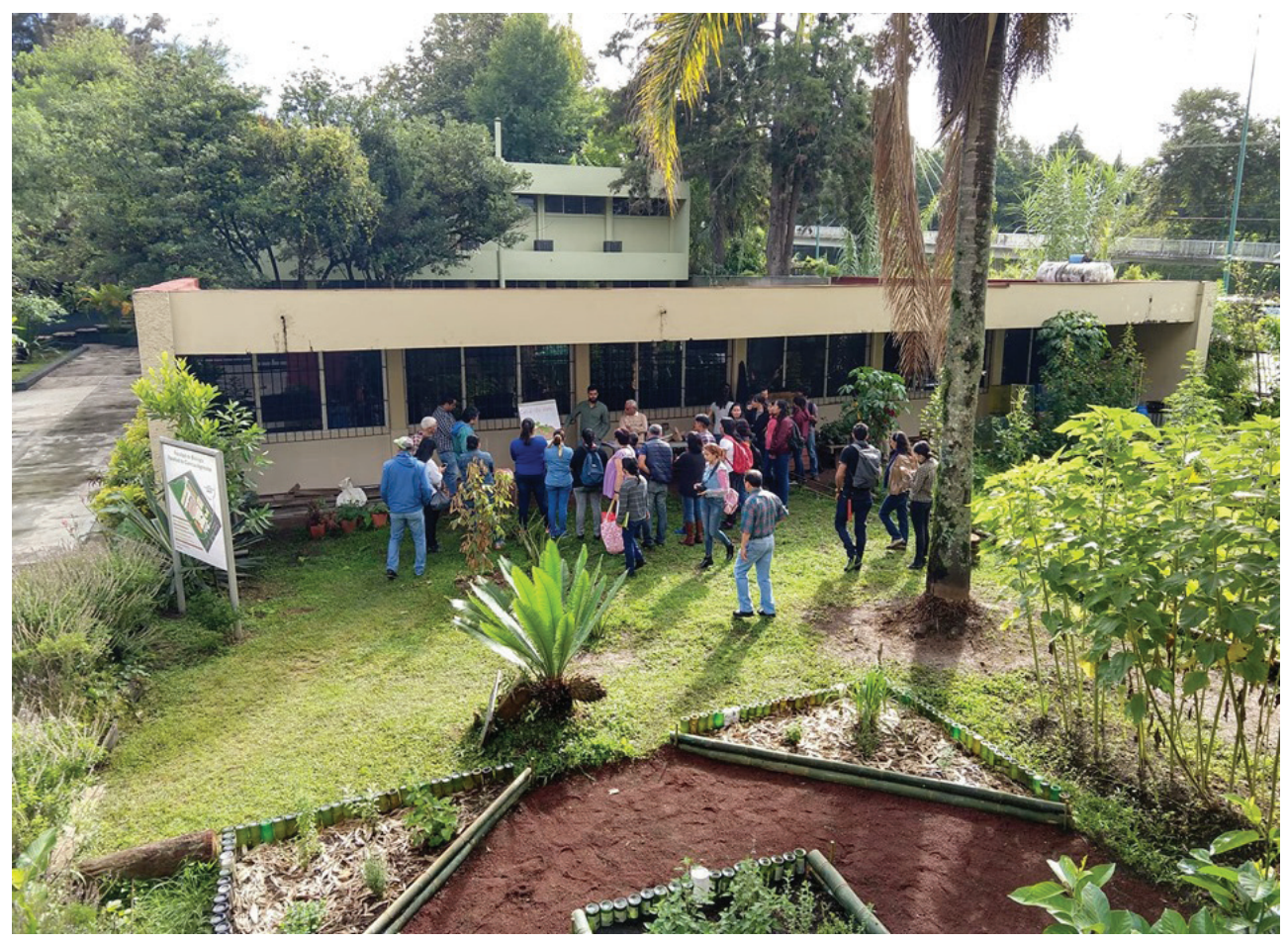

Figura $\mathbf{N}^{\circ}$ 1: Juan Fontalvo (2020) 


\subsection{Tipo de investigación}

Según la profundidad del objeto de estudio, esta es una investigación exploratoria que pretende ofrecer algunas nociones generales acerca de los huertos como satisfactores de necesidades humanas fundamentales. Este tipo de investigación es utilizada para estudiar un problema que no está claramente definido, por lo que se lleva a cabo para comprenderlo mejor, pero sin proporcionar resultados concluyentes (Kothari, 2004). Según la naturaleza de los datos, este es un trabajo de investigación cualitativa. En este proceso, se toman en cuenta los discursos de los sujetos y las conductas observables en un tiempo determinado desde diferentes instrumentos (Rodríguez et al., 1996) para luego interpretarlos, analizarlos y relacionarlos, en este caso, dentro de un contexto social, educativo y de comunidad. Esta aproximación bibliográfica y vivencial se realizó siguiendo la metodología de estudio de caso propuesta por Hamel y colaboradores (1993), que distingue cuatro momentos: formulación de la pregunta de reflexión, definición de la unidad de análisis, instrumentos para la recolección de información y análisis de la información.

\subsection{Pregunta de reflexión}

¿De qué manera el Huerto Agroecológico y sus actividades asociadas pueden ser un espacio o canal para satisfacer necesidades humanas fundamentales de los miembros de la Facultad de Biología de la Universidad Veracruzana?

\subsection{Unidad de análisis}

Miembros de la Facultad de Biología en la región Xalapa y de la red de huertos de la Universidad Veracruzana que han asistido y/o participado en las actividades del Huerto Agroecológico entre enero de 2014 y junio de 2021, períodos en los que la Facultad de Biología ha estado conformada con una población promedio aproximada de 700 personas, en la que la mayoría (85 \%) eran estudiantes, y el resto (15\%), académicos y personal de apoyo (jardineros, aseadores, secretarias, administrativos y vigilantes).

\subsection{Instrumentos para la recolección de información}

El aspecto bibliográfico se desarrolló a partir del método de búsqueda especializada propuesto por Tranfield y colaboradores (2003), el cual contempla tres etapas principales: (1) la planificación detallada a priori de la búsqueda con una definición precisa del objetivo de investigación; (2) una ejecución rigurosa de la búsqueda, que comprende la identificación de literatura relevante usando palabras clave relacionadas con la temática y operadores booleanos; y (3) el análisis e integración de la información al estudio. El aspecto vivencial se sistematizó a partir de la técnica de observación participante durante la intervención de los miembros de la comunidad en diferentes actividades relacionadas con el Huerto Agroecológico, tales como clases, prácticas escolares, tequios, cursos, charlas, entre otras modalidades participativas. Durante la observación documental, se tomaron en cuenta los discursos de los sujetos, así como sus actitudes, comportamientos y conductas observables (Bravo, 1995). 


\subsection{Análisis de la información}

La información sistematizada se analizó considerando las necesidades fundamentales y las categorías existenciales del ser, tener, hacer y estar (Max-Neef, 1992). De forma concreta, se analizó cada categoría para identificar patrones significativos y luego fueron sustentados teóricamente con la información obtenida en la búsqueda especializada.

\section{Resultados}

Para efectos de esta aproximación, retomamos las nociones necesidad y capacidad de forma indistinta, teniendo en cuenta que, de una forma $\mathrm{u}$ otra, tienen su incidencia en el desarrollo humano y el bienestar social. Sin embargo, hemos encontrado gran afinidad para asociar el huerto universitario con los criterios de necesidades humanas definidas por Max-Neef (1992). Esta relación está dada por la versatilidad y multifuncionalidad de estos espacios, pero también por sus beneficios emergentes en los contextos educativos, lo cual, de manera conjunta, permite que tengan incidencia positiva en las categorías del ser, tener, hacer y estar. De forma general, consideramos que el huerto es un importante medio para desarrollar y/o cumplir necesidades humanas fundamentales (Tabla 1).

Tabla 1. Necesidades humanas que se pueden satisfacer en huertos universitarios.

\begin{tabular}{|c|c|c|c|c|}
\hline Necesidad & $\begin{array}{c}\text { Ser } \\
\text { (Características) }\end{array}$ & $\begin{array}{c}\text { Tener } \\
\text { (Herramientas) }\end{array}$ & $\begin{array}{c}\text { Hacer } \\
\text { (Acciones) }\end{array}$ & $\begin{array}{c}\text { Estar } \\
\text { (Espacio) }\end{array}$ \\
\hline Subsistencia & Salud física y mental & Alimentación & Comer y descansar & \multirow{9}{*}{$\begin{array}{l}\text { Huerto } \\
\text { Agroecológico }\end{array}$} \\
\hline Protección & Cuidado y adaptabilidad & Seguridad social & $\begin{array}{c}\text { Cooperar, planificar } \\
\text { y ayudar }\end{array}$ & \\
\hline Afecto & $\begin{array}{l}\text { Respeto, sentido del } \\
\text { humor, generosidad }\end{array}$ & $\begin{array}{c}\text { Amistades y relaciones } \\
\text { con la naturaleza }\end{array}$ & $\begin{array}{l}\text { Compartir, cuidar, } \\
\text { expresar emociones }\end{array}$ & \\
\hline Entendimiento* & $\begin{array}{c}\text { Capacidad crítica, curiosidad } \\
\text { e intuición }\end{array}$ & Educación & $\begin{array}{l}\text { Analizar, estudiar, } \\
\text { meditar e investigar }\end{array}$ & \\
\hline Participación* & $\begin{array}{l}\text { Receptividad } \\
\text { y dedicación }\end{array}$ & $\begin{array}{l}\text { Responsabilidades } \\
\text { y deberes }\end{array}$ & $\begin{array}{l}\text { Cooperar y expresar } \\
\text { opiniones }\end{array}$ & \\
\hline Ocio* & Tranquilidad y espontaneidad & Paz mental & Relajarse y divertirse & \\
\hline Creación & $\begin{array}{l}\text { Imaginación, inventiva } \\
\text { y curiosidad }\end{array}$ & $\begin{array}{l}\text { Capacidades } \\
\text { y habilidades }\end{array}$ & $\begin{array}{l}\text { Inventar, construir, } \\
\text { diseñar, componer e } \\
\text { interpretar }\end{array}$ & \\
\hline Identidad* & $\begin{array}{c}\text { Sentido de pertenencia } \\
\text { y autoestima }\end{array}$ & Principios y valores & Crecer y comprometerse & \\
\hline Libertad & $\begin{array}{l}\text { Autonomía y } \\
\text { mente abierta }\end{array}$ & Igualdad de derechos & $\begin{array}{l}\text { Disentir, escoger, } \\
\text { concienciarse }\end{array}$ & \\
\hline
\end{tabular}

Fuente: Adaptado a partir de Max-Neef (1992).

Nota: ${ }^{*}$ Necesidades humanas que satisface el huerto universitario en mayor proporción.

De manera directa y en mayor proporción, las necesidades de ocio, entendimiento, participación e identidad, y, en menor medida, las de subsistencia, protección, afecto, creación y libertad. Lo más rescatable del huerto universitario en este ámbito es que se trata de un satisfactor sinérgico de necesidades humanas fundamentales; esto es, estimula 
y contribuye a la satisfacción simultánea de varias necesidades. Por todo lo anterior, estos espacios deben ser muy valorados, debido a que pocos canales o entornos logran tener este efecto sinérgico entre las necesidades (Morán, 2014).

En primer lugar, el huerto es un medio idóneo para cubrir la necesidad de ocio en las personas (Núñez et al., 2009). Durante los tequios o reuniones periódicas, los participantes así lo suelen manifestar. Las personas involucradas definen el huerto como un espacio en el que se sienten relajadas y pueden divertirse aprendiendo. Esto se debe a que el huerto siempre está abierto, es próximo a las aulas, y es un área verde que promueve y desarrolla la imaginación y la espontaneidad, al mismo tiempo que permite a las personas gastar su tiempo libre y aprender (Santa-Cruz, 2015). Además, hemos apreciado que los estudiantes y, mayormente, el personal de apoyo utilizan el huerto para pasar sus tiempos libres, con lo cual obtienen esparcimiento y relajación ya sea contemplando el paisaje del huerto con sus componentes, deshierbando las camas de cultivo, cosechando las hortalizas o las plantas aromáticas y medicinales.

En segundo lugar, el huerto es un canal importante para promover la capacidad de entendimiento. Esta es quizá la necesidad con la que más se lo asocia, porque los huertos son muy reconocidos por servir como herramienta de enseñanza (Ruiz y Rodríguez, 2015). La mayoría de los docentes han encontrado en los huertos un "aula-laboratorio" para orientar algunos temas relacionados con sus experiencias educativas y desarrollar procesos experimentales como prácticas escolares e investigaciones. Estos espacios también están siendo utilizados como un instrumento de educación ambiental que permite realizar extensión universitaria y sensibilizar acerca de la soberanía alimentaria, la separación de residuos, el cuidado de la naturaleza y el desarrollo de prácticas sustentables a través de la ecoformación (Barrón y Muñoz, 2015). De forma general, los huertos tienen componentes importantes para generar las capacidades de criticar, analizar y meditar, pero también pueden promover la curiosidad y la intuición en sus participantes. Por ejemplo, a través del huerto, los estudiantes de la licenciatura en Biología ofrecen recorridos guiados a niños de escuelas de educación básica, mientras que los docentes instruyen a adultos para construir huertos caseros. En ambos casos, se enseña a cuidar el medio ambiente. Particularmente, se busca que, en estos casos, las actividades desarrolladas en el huerto universitario puedan conducir a los estudiantes, mediante la práctica, a adoptar hábitos sustentables y comportamientos ambientales responsables (Acuña y Sánchez, 2019).

En tercer lugar, los huertos son espacios que favorecen la capacidad de participación debido a su enfoque comunitario. Esto permite a los actores cooperar, disentir, expresar opiniones y ejercer democracia sobre decisiones y procesos por desarrollar en el huerto; en otras palabras, el huerto es un espacio de participación por naturaleza, es su razón de ser (del Viso et al., 2017). Esto resulta valioso porque, de esta manera, se contrarresta la actitud pasiva de los estudiantes en los momentos pedagógicos y permite que puedan tener un rol más protagónico en los procesos de aprendizaje (Larson, 2015). Hemos visto la forma en que los docentes se ayudan de los componentes del huerto para orientar las asignaturas básicas de la licenciatura en Biología, así como ejes transversales de la educación superior. El ambiente pedagógico que generan estos espacios cultivados ayuda a que las clases sean más didácticas, que los estudiantes participen más y que los objetivos de aprendizaje sean 
alcanzados (Fontalvo-Buelvas, 2021). Además, las reuniones colectivas en el huerto han propiciado procesos participativos que no solo involucran a docentes y estudiantes, sino también a secretarias, aseadores, jardineros y otros miembros del personal de apoyo, una integración comunitaria que se visualiza poco en otras facultades de la universidad.

En cuarto lugar, el huerto promueve actividades de autogestión, apropiación e intervención que involucran diseńo, siembra, mantenimiento, cosecha, intercambio y consumo, procesos que son realizados mediante tequios, una serie de trabajos colectivos en beneficio de la comunidad. Además, de forma transversal durante estas faenas, se incentiva la democracia, dando la oportunidad de tomar decisiones consensuadas; el debate, procurando el intercambio de pensamientos; y la libertad, ofreciendo la apertura para que cada participante actúe según sus convicciones. En este caso, la libertad también se ve estimulada cuando se respetan las voluntades individuales de participación, y cada miembro de la comunidad elige los roles y responsabilidades que aspira asumir (Aznar, 2014; Mundel y Chapman, 2010). Además, el huerto promueve la libertad desde su estructura física, ya que no tiene puertas; por tanto, su entrada siempre está abierta y todos los miembros de la comunidad son libres de ingresar y participar. Justamente, esa estructura ha permitido que cada vez se vayan sumando más miembros de la comunidad universitaria a las actividades del huerto, en modalidades como el servicio social y el voluntariado.

En quinto lugar, el huerto es un medio para generar identidad, tanto a nivel colectivo como individual (Wen Li et al., 2010). Las personas que participan periódicamente en estos espacios se sienten identificadas con una serie de acciones sustentables, es decir, un conjunto de hábitos y costumbres que contribuyen al bienestar social y al cuidado del medio ambiente. De igual manera, experimentan un sentido de pertenencia hacia la naturaleza y se comprometen con ella gracias a los valores, principios y actitudes adquiridas durante las faenas grupales. Cuando un colectivo se reúne con frecuencia en un lugar determinado, se empiezan a desarrollar vínculos sólidos entre las personas y entre estas con el lugar (Flap y Völker, 2005; Völker et al., 2007). Esto permite que se cubran, de forma progresiva y simultánea en el tiempo, las necesidades de identidad, participación, afecto, libertad, entendimiento y protección; a esto se le llama efecto sinérgico de un canal de satisfacción. En el caso del huerto universitario, cada año se va afianzando más la identidad que las personas sienten con este lugar gracias a la constancia de las actividades realizadas, los procesos de difusión permanente y, particularmente, debido al tejido social que se ha construido.

En sexto lugar, el huerto puede ser un canal para suscitar levemente la necesidad de protección y, así, reducir la vulnerabilidad social (Gutiérrez-Cedillo et al., 2018). Esto es posible en la medida en que estos espacios puedan suministrar condiciones de estancias agradables, en las que las personas se sientan a gusto para el esparcimiento y la recreación, pero, sobre todo, para huir del caos que representa la ciudad, o el estrés universitario y laboral. Para propiciar este escenario es indispensable contar con un diseño de huerto agradable (Oliveira, 2010), que contemple laberintos de hierbas aromáticas, plantas con flores todo el año y áreas para sentarse. Es indispensable que el huerto no deje de ser un espacio de participación y de identidad comunitaria, pues esto influirá en que las 
personas se sientan seguras (Barthel e Isendahl, 2013). Por ejemplo, el huerto cuenta con la presencia de una gran variedad de árboles, arbustos y hierbas en oposición al resto de espacios comunes estériles de la facultad. Esto, con frecuencia, atrae a los miembros de la comunidad y conduce a contactos más frecuentes entre las personas (Coley et al., 1997). Los huertos, como áreas cultivadas dentro del espacio común de la universidad, proporcionan sombra y amortiguan el sonido de los entornos circundantes, por lo que podrían tener efectos restauradores para el paisaje y la comunidad universitaria (Hartig et al., 2003; Kaplan y Kaplan, 1989), pues este tipo de áreas verdes contribuye en la regulación de la temperatura y la purificación del aire, al tiempo que tienen valor estético y ofrecen espacio para el esparcimiento.

En séptimo lugar, el huerto es un entorno propicio para desplegar la capacidad de creatividad, ya que la mayoría de las actividades que se llevan a cabo en estos espacios exigen destreza e imaginación (Watson-Soto et al., 2010). Usualmente, el diseño del huerto, la siembra, el riego, la poda, la cosecha, y, particularmente, el manejo de plagas y enfermedades, son labores que requieren destreza, ingenio y originalidad. De manera general, los huertos y sus componentes promueven la curiosidad e incitan a descubrir nombres, formas, colores, procesos, fenómenos, recetas, relaciones, etc. Estos son diferentes aspectos didácticos de los huertos que se aprovechan durante los momentos pedagógicos para construir actividades dinámicas, generar aprendizajes y desarrollar habilidades del pensamiento crítico (Moromizato, 2007). Por ejemplo, es frecuente ver a estudiantes realizando ilustraciones de las plantas, los polinizadores y otros insectos del huerto universitario; algunos lo hacen como parte de sus responsabilidades, aunque también hay quienes lo hacen de forma espontánea como una actividad de relajación.

Se ha mencionado que la satisfacción de afecto se consigue exclusivamente a través de espacios íntimos (Max-Neef, 1992), especialmente porque se relaciona con el afecto de pareja. Sin embargo, es usual que las personas que participan en el huerto empiecen a establecer vínculos afectivos interpersonales como la amistad y vínculos con la naturaleza como la empatía ambiental. Principalmente, los estudiantes encuentran en el huerto un espacio para compartir, expresar emociones y construir lazos de hermandad con compañeros de diferentes semestres gracias a las actividades en las que participan. Esto también influye en la forma en que se relacionan con el medio ambiente, ya que, gracias al contacto diario que tienen con los organismos vivos, se propicia la creación de hábitos socioambientales positivos. Cuando se tiene vínculos afectivos con los demás, se empieza a sentir una conexión con dicho lugar. Esto se traduce en la identidad de la comunidad (Prezza et al., 2001). Según Kim y Kaplan (2004), el sentido de comunidad de las personas que comparten espacios con frecuencia se fortalece cuando se mantiene la frecuencia con la que se reúnen. Particularmente, el colectivo del huerto tiene por costumbre reunirse mensualmente. En diversas ocasiones, los estudiantes han manifestado que continúan participando como voluntarios en el huerto debido a los vínculos afectivos generados no solo con los miembros del colectivo, sino también con el lugar.

Finalmente, el huerto puede responder levemente a la necesidad de subsistencia, respecto de la alimentación y la salud (Martínez y Juan, 2005). Aunque los huertos carecen de la capacidad para abastecer de alimentos a la totalidad de la comunidad 
universitaria, en general, sí producen un pequeño grupo de hortalizas, frutos y plantas medicinales que tienen el valor agregado de ser productos ecológicos y saludables. Así, estas iniciativas permiten que las personas construyan huertos en sus casas o bien empiecen a comprar en mercados agroecológicos, ambos aspectos que aportan a la salud de la comunidad. Por otro lado, es indispensable que los colectivos de personas asociadas a los huertos universitarios opten por opciones innovadoras como la agroecología (Altieri et al., 1999), la permacultura (Muñiz, 2019) o la agricultura orgánica (Suquilanda, 1996). En otras palabras, no utilizar pesticidas, ni semillas modificadas genéticamente u otras prácticas convencionales para asegurar la salud del agroecosistema (Sarandón y Flores, 2014). También, se aporta a la soberanía y seguridad alimentaria de la comunidad universitaria al tiempo que se promueve una alimentación saludable (Trescastro-López y Trescastro-López, 2013). Por ejemplo, el huerto provee a la cafetería de la facultad de insumos como hortalizas y hierbas finas a cambio de que esta deposite diariamente sus residuos orgánicos en las compostas del huerto. De esta manera, se ofrecen algunos platillos saludables para la comunidad universitaria, a la vez que se va produciendo abono orgánico para las plantas. Esta y otras buenas prácticas han permitido que la universidad le otorgue el distintivo "cafetería sustentable", un reconocimiento por su contribución a la sustentabilidad universitaria.

\section{Discusión}

\section{1. Beneficios derivados de la satisfacción de necesidades}

Teniendo en cuenta las necesidades humanas fundamentales en las que el huerto puede ser un medio importante para satisfacer en pequeña o gran medida, es posible resaltar que estos espacios en el contexto universitario poseen un considerable potencial para generar una serie de efectos positivos sobre el bienestar social (Romá-Ferri et al., 2017). Esto se encuentra asociado al valor que supone para las personas tener acceso a alimentos frescos, ecológicos y de calidad en espacios próximos, y que, además, son sembrados y cosechados por los mismos estudiantes, docentes y personal de la comunidad universitaria (Morán, 2009). Asimismo, el huerto universitario puede contribuir a la reconstrucción de condiciones sociales que generan una mayor resiliencia en la comunidad universitaria (Barthel et al., 2015). Sea entendida la resiliencia en este escenario como una mayor capacidad de la comunidad para responder a desafíos o impactos externos sin que su organización académica interna se vea afectada. Por ejemplo, en situaciones de dificultades causadas por factores como el estrés, la sobrecarga de responsabilidades o el rezago social, el huerto universitario puede favorecer el esparcimiento, la relajación y el contacto con la naturaleza para contrarrestar las presiones académicas y sociales que comúnmente se viven en los entornos universitarios. Estas áreas verdes permiten educar para la vida al mismo tiempo que contribuyen en la consecución de entornos más inclusivos, accesibles y sustentables, una de las grandes metas de las sociedades modernas (Ferris et al., 2001).

Las áreas verdes y los huertos deben ser considerados como espacios prioritarios para el contexto urbano y el bienestar humano (Daily, 1997). Estos espacios prestan 
servicios ecosistémicos como la mitigación de la contaminación del aire, el ruido y el calor, y proveen espacio para recreación y educación (McGranahan et al., 2005). Dada la relevancia de las interacciones entre el huerto y los seres humanos, es necesario comprender mejor cómo interactúan los factores sociales con paisajes urbanos para producir dinámicas socioecológicas (Grimm et al., 2000), especialmente porque estas áreas verdes sufren una gran presión, ya que la mancha urbana y su infraestructura (estructuras, redes y servicios) se extiende y evoluciona a gran velocidad, lo que disminuye drásticamente los servicios ecosistémicos de los espacios naturales (Artasu, 2016). Por ejemplo, la Organización Mundial de la Salud sugiere entre 9 y $11 \mathrm{~m}^{2}$ de área verde por habitante para garantizar el derecho a vivir en un ambiente sano (ONU-Hábitat, 2016). Las áreas verdes son un indicador de sustentabilidad urbana y calidad de vida, por lo que deben promoverse con mayor ímpetu desde la planificación territorial (Shen et al., 2011). En el caso de los huertos, estos deben articularse a la agricultura urbana y periurbana que incluye huertos individuales y colectivos, la cual es un fenómeno que resignifica la relación entre las ciudades y el campo (Bórquez y Ventura, 2009). Este tipo de espacios citadinos están compuestos de valiosas cualidades socioecológicas debido a las diferentes prácticas de gestión y participación; sin embargo, un aspecto singular es el hecho de que promueven vínculos fuertes entre las personas, el medio ambiente, la alimentación y la salud (Specht et al., 2014).

\section{2. Recomendaciones para mantener la capacidad de satisfactor}

Para mantener o potenciar la capacidad de los huertos universitarios como satisfactores de las necesidades humanas fundamentales, es necesaria una gestión integral. Esta gestión debe concebir un manejo adecuado del agroecosistema o área verde, y un enfoque participativo en todos los procesos y actividades por desarrollarse (Fernández y Morán, 2012). Para el manejo, se recomienda seguir los principios de la agroecología (Altieri y Nicholls, 2013), ya que este enfoque productivo persigue la sostenibilidad de los agroecosistemas (Gliessman et al., 2007). En este sentido, ambientes sostenibles y saludables proporcionan más y mejores servicios ecosistémicos (Caro-Caro y Torres-Mora, 2015), entre ellos, el de favorecer de forma óptima la satisfacción de las necesidades humanas fundamentales. Para el enfoque participativo, se recomienda el desarrollo de actividades tanto grupales e individuales basadas en el diálogo de saberes (Richard y Contreras, 2013) y el aprendizaje colectivo (Fam et al., 2018). La asignación de roles y deberes crea compromisos y responsabilidades, pero, sobre todo, permite el desarrollo organizativo (Mazzotti y Nava, 2019), el fortalecimiento de lazos sociales y la consolidación del tejido social resiliente (Peredo-Parada et al., 2016). Es indispensable que las actividades que se realizan en el huerto universitario sean respetuosas con el ambiente para así propiciar conductas socioambientales positivas en los involucrados (Pérez-González, 2018).

\section{Conclusiones}

En definitiva, el huerto universitario es un medio a través del cual se pueden cubrir, desarrollar o potenciar de forma sinérgica las necesidades humanas fundamentales. En este sentido, es importante recalcar que los huertos no satisfacen necesidades humanas 
por sí mismos. Dichas áreas verdes cultivadas son apenas un canal para lograr tal fin, por lo que estos procesos dependen del uso y manejo que las personas y colectivos les otorgan. Los huertos en el contexto universitario tienen una dinámica compleja con interacciones sociales, bienes y servicios que posibilitan en diferentes proporciones la satisfacción de necesidades humanas fundamentales. Este tipo de procesos tiene una gran incidencia en el desarrollo personal y comunitario, lo que se traduce en una mejora del bienestar de las comunidades universitarias. Por lo tanto, resulta conveniente incentivar los huertos e institucionalizarlos desde políticas internas para que sean parte orgánica de los campus, se articulen a los ejes transversales y contribuyan a la sustentabilidad universitaria.

Por otro lado, el enfoque agroecológico asociado a las prácticas de manejo y el fundamento comunitario vinculado a sus actividades les otorgan a los huertos universitarios una esencia crucial para la satisfacción de las necesidades humanas. Lo primero permite que estos espacios sean ambientalmente responsables, y lo segundo hace posible que sean socialmente aceptables y culturalmente significativos. El diálogo, la cooperación y la identidad colectiva son algunos procesos clave que facilitan o detonan el alcance de las necesidades. Por tanto, resulta imprescindible que los colectivos asociados a los huertos logren un buen engranaje socio-ecológico para mantener en el tiempo no solo la salud del agroecosistema, sino también la capacidad de este para proveer efectos sinérgicos.

Por último, es importante puntualizar que los aspectos desglosados son apenas una aproximación bibliográfica y vivencial construida a partir de las necesidades humanas fundamentales descritas por Max-Neef. Esta conjetura puede representar un punto de partida importante para una discusión más profunda sobre la relación existente entre los huertos y las necesidades o capacidades humanas. Evidentemente, en un siguiente momento, será necesario visualizar aquellas herramientas metodológicas que permitan identificar y evaluar los procesos y fenómenos sociales aquí suscitados.

\section{Referencias bibliográficas}

Acuña, B. y Sánchez, C. (2019). El huerto escolar: estrategia didáctica para la promoción de valores ambientales en la educación inicial. Revista de Propuestas Educativas, 1(2), 95-113.

Aldea, E. (2012). El huerto escolar como recurso educativo de centros de educación [Tesis de maestría en Educación]. La Rioja, España: Universidad Internacional de La Rioja.

Altieri, M., Hecht, S., Matt, L., Fred, M., Richard, N. y Thomas, S. (1999). Agroecología: bases cientificas para una agricultura sustentable. Nordan-Comunidad.

Altieri, M. y Nicholls, C. (2013). Agroecología y resiliencia al cambio climático: principios y consideraciones metodológicas. Agroecología, 8(1), 7-20.

Álvarez-Ferri, S. (2012). Huertos orgánicos como instrumento estratégico para la regeneración urbana en Canarias: Proyecto Verde Cooperativo de Regeneración Urbana en el Polígono Residencial de Arinaga, Agüimes [Tesis de maestría en Energías Renovables. Arquitectura y Urbanismo]. España: Universidad Internacional de Andalucía.

Artasu, M. C. (2016). Las áreas verdes en la Ciudad de México. Las diversas escalas de una geografía urbana. Biblio3W Revista Bibliográfica de Geografía y Ciencias Sociales, 21(1), 1-22. 
Aznar, I. (2014). El huerto urbano como herramienta para la formación de personas en situación de exclusión social [Tesis de licenciatura en Ciencias Ambientales]. Espańa: Universidad Politécnica de Valencia.

Barrón, Á. y Muñoz, J. (2015). Los huertos escolares comunitarios: fraguando espacios socioeducativos en y para la sostenibilidad. Foro de Educación, 13(19), 213-239.

Barthel, S., Parker, J. y Ernstson, H. (2015). Food and green space in cities: A resilience lens on gardens and urban environmental movements. Urban Studies, 52(7), 13211338.

Barthel, S. e Isendahl, C. (2013). Urban gardens, agriculture, and water management: Sources of resilience for long-term food security in cities. Ecological Economics, 86, 224-234.

Bórquez, L. C. y Ventura, S. G. (2009). Nueva ruralidad y desarrollo territorial. Una perspectiva desde los sujetos sociales. Veredas: Revista del Pensamiento Sociológico, 1(18), 145-167.

Botella, N., Amparo, H. y Cantó, J. (2017). El huerto escolar como herramienta innovadora que contribuye al desarrollo competencial del estudiante universitario. Una propuesta educativa multidisciplinar. Vivat Academia, 139(1), 19-31.

Bravo, R. (1995). Técnicas de investigación social: teoría y ejercicios. Editorial Paraninfo.

Camps-Calvet, M., Langemeyer, J., Calvet-Mir, L. y Gómez-Baggethun, E. (2016). Ecosystem services provided by urban gardens in Barcelona, Spain: Insights for policy and planning. Environmental Science \& Policy, 62, 14-23.

Caro-Caro, C. y Torres-Mora, M. (2015). Servicios ecosistémicos como soporte para la gestión de sistemas socioecológicos: aplicación en agroecosistemas". Orinoquia, 19(2), 237-252.

Coley, R., Sullivan, W. y Kuo, M. (1997). Where does community grow? The social context created by nature in urban public housing. Environment and behavior, 29(4), 468-494.

Daily, G. (ed.) (1997). Nature's services: societal dependence on natural ecosystems. Island Press.

Deelstra, T. (1987). Urban agriculture and the metabolism of cities". Food and Nutrition Bulletin, 9(2), 1-3.

Del Viso, N., Fernández, J. y Morán, N. (2017). Cultivando relaciones sociales. Lo común y lo "comunitario" a través de la experiencia de dos huertos urbanos de Madrid. Revista de Antropología Social, 26(2), 449-472.

Doyal, L. y Gough, I. (1991). A theory of human need. Guilford Press.

Eugenio-Gozalbo, M. y Aragón-Núnez, L. (2016). Experiencias en torno al huerto ecológico como recurso didáctico y contexto de aprendizaje en la formación inicial de maestros de Infantil. Revista Eureka sobre Enseñanza y Divulgación de las Ciencias, 13(3), 667-679.

Fam, D., Neuhauser, L. y Gibbs, P. (eds.) (2018). Transdisciplinary theory, practice and education. Springer International Publishing AG.

Fernández, J. y Morán, N. (2012). Nos plantamos! Urbanismo participativo y agricultura urbana en los huertos comunitarios de Madrid. Hábitat y sociedad, 4(4), 55-71. 
Ferris, J., Norman, C. y Sempik, J. (2001). People, land and sustainability: Community gardens and the social dimension of sustainable development. Social Policy \& Administration, 35(5), 559-568.

Flap, H. y Völker, B. (2005). Gemeenschap, informele controle en collectieve kwaden in de buurt. En B. Völker (ed.), Burgers in de buurt: samenleven in school, wijk en vereniging ( $p$ p. 41-70). Amsterdam University Press.

Fontalvo-Buelvas, J. C. (2021). El Huerto Agroecológico y sus procesos sustentables para apoyar las funciones sustantivas de la Facultad de Biologia-Xalapa, Veracruz [Tesis de maestría]. México: Universidad Veracruzana.

Gliessman, S., Rosado-May, F., Guadarrama-Zugasti, C., Jedlicka, J., Cohn, A., Méndez, E. y Jaffe, R. (2007). Agroecología: promoviendo una transición hacia la sostenibilidad. Revista Ecosistemas, 16(1), 13-23.

Grimm, N., Grove, G., Pickett, S. y Redman, C. (2000). Integrated approaches to longterm studies of urban ecological systems. Bioscience, 50(7), 571-583.

Groppa, O. (2005). Las necesidades humanas y su determinación: los aportes de Doyal y Gough, Nussbaum y Max-Neef al estudio de la pobreza. Instituto para la Integración del Saber, Universidad Católica Argentina.

Gutiérrez-Cedillo, J., García-Flores, J. y Franco-Roberto, P. (2018). Los huertos familiares como reguladores de la vulnerabilidad socioambiental y activadores de la resiliencia territorial. Ciencias y Medio Ambiente, 18(1), 30-37.

Hamel, J., Dufour, S. y Fortin, D. (1993). Case study methods. Sage Publications.

Hartig, T., Evans, G., Jamner, L., Davis, D. y Gärling, T. (2003). Tracking restoration in natural and urban field settings. Journal of environmental psychology, 23(2), 109-123.

Henríquez-Henríquez, C., Watson-Soto, H. y Camacho-Brown, L. (2010). Lo lúdico creativo al encuentro del huerto escolar. Universidad de Costa Rica-Vicerrectoría de Acción Social.

Hilerio-Cruz, J. (2017). El huerto, proyecto agroalimentario con niños de preescolar [Tesis de Licenciatura en Ingeniería Agroalimentaria]. México: Universidad de Ciencias y Artes de Chiapas.

Moromizato, R. K. (2007). El desarrollo del pensamiento crítico creativo desde los primeros años. El ágora USB, 7(2), 311-321.

Kaplan, R. y Kaplan, S. (1989). The experience of nature: A psychological perspective. Cambridge University Press.

Kim, J.y Kaplan, R. (2004). Physical and psychological factors in sense of community: New urbanist Kentlands and nearby Orchard Village. Environment and behavior, 36(3), 313-340.

Kothari, C. R. (2004). Research methodology: Methods and techniques. New Age International.

Lafuente-Miranda, S. (2017). El huerto escolar: un espacio de enseñanza-aprendizaje de las ciencias de la naturaleza en Educación Infantil [Tesis de Licenciatura en Educación Infantil]. España: Universidad de Valladolid. 
López, M., Hervías, E., Molina, L., Ortega, S., Jiménez, I., García, J., Romero, M. y Guzmán, M. (2011). Huerto ecológico y vivero escolar; algo más que un recurso educativo. Revista Digital Educativa Wadi-red, 1(1), 39-49.

Larson, N. (2015). La enseñanza en el aula de la naturaleza. Principios fundamentales del aprendizaje en los huertos educativos. Environmental Design Lab Press, Madison.

Maas, J., van Dillen, S., Verheij, R. y Groenewegen, P. (2009). Social contacts as a possible mechanism behind the relation between green space and health. Health \& place, 15(2), 586-595.

Malca, N. (2012). Contribución de las áreas verdes urbanas a la calidad ambiental del distrito de Comas-Lima, al año 2011. Revista del Instituto de Investigación de la Facultad de Ingeniería Geológica, Minera, Metalúrgica y Geográfica, 15(30), 117-121.

Martínez, R. y Juan, J. (2005). Los huertos: una estrategia para la subsistencia de las familias campesinas. Anales de Antropología, 39(2), 25-50.

Martínez-Soto, J., Montero, M. y de la Roca-Chiapas, J. (2016). Efectos psicoambientales de las áreas verdes en la salud mental. Interamerican journal of psychology, 50(2), 204214.

Maslow, A. (1943). A theory of human motivation. Psychological review, 50(4), 370-396. Max-Neef, M. (1992). Development and human needs. En P. Ekins y M. Max-Neef (eds.), Real-life economics: Understanding wealth creation (pp. 197-213). Routledge.

Max-Neef, M. (1993). Desarrollo a escala humana: Conceptos, aplicaciones y reflexiones. Icaria Editorial.

McGranahan, G., Marcotullio, P., Bai, X., Balk, D., Braga, T., Douglas, I., Elmqvist, T., Rees, W., Satterthwaite, D., Songsore, J., Eades, J. y Ezcurra, E. (2005). Urban systems. En R. Hassan, R. Scholes y N. Ash (eds.), Ecosystems and Human Well-being: Current State and Trends (pp. 795-825). Island Press.

Merçon, J., Escalona-Aguilar, M., Noriega-Armella, I., Figueroa-Núńez, I., AtencoSánchez, A. y González-Méndez, E. (2012). Cultivando la educación agroecológica: el huerto colectivo urbano como espacio educativo. Revista mexicana de investigación educativa, 17(55), 1201-1224.

Moctezuma-Pérez, S. (2010). Una aproximación al estudio del sistema agrícola de huertos desde la Antropología. Ciencia y Sociedad, 35(1), 47-69.

Montagnini, F. y Metzel, R. (2015). Biodiversidad, manejo de nutrientes y seguridad alimentaria en huertos caseros mesoamericanos. En F. Montagnini, E. Somarriba, H. Fassola y B. Eibl (eds.), Sistemas agroforestales. Funciones productivas, socioeconómicas y ambientales (pp. 381-404). Editorial CIPAV.

Morán, N. (2009). Huertos y jardines comunitarios. Boletín $C F+S$, 4O(1), 1-23.

Morán, N. (2014). Huertos urbanos en tres ciudades europeas: Londres, Berlín, Madrid. Boletin $C F+S, 47 / 48(1), 75-124$.

Mundel, E. y Chapman, G. (2010). A decolonizing approach to health promotion in Canada: the case of the Urban Aboriginal Community Kitchen Garden Project. Health Promotion International, 25(2), 166-173.

Muñiz, R. (2019). El aporte de la permacultura en el desarrollo de la agricultura urbana. Tekhné, 22(1), 35-43. 
Núñez, M., Borrella, F. y Durán, G. (2009). Una experiencia de huerto escolar enmarcada en huertos de ocio intergeneracionales. Aula de innovación educativa, 183(1), 44-47.

Nussbaum, M. C. (1998). Virtudes no relativas: un enfoque aristotélico. En M. C. Nussbaum, Y A. Sen (Eds.). La calidad de vida (pp. 318-351). Fondo de Cultura Económica.

Oliveira, M. (2010). Jardines coloniales brasileños: sitios de lo útil a lo agradable. Norba: revista de arte, 30(1), 247-259.

ONU-Hábitat. (2016). La iniciativa de la prosperidad urbana. Naciones Unidas.

Mazzotti, G y Nava, K. (2019). Comunalidad y transmodernidad en las prácticas organizativas de la red de huertos educativos en México. REAd. Revista Eletrônica de Administração (Porto Alegre), 25(3), 203-222.

Peredo-Parada, S., Vela-Campoy, M. y Jiménez-Gómez, A. (2016). Determinación de los niveles de resiliencia/vulnerabilidad en iniciativas de agroecología urbana en el suroeste andaluz. Idesia (Arica), 34(2), 5-13.

Pérez-González, D. (2018). La apropiación de las áreas comunes urbanas y sus implicaciones socioambientales [Tesis de Maestría en Ciencias Ambientales]. México: Benemérita Universidad Autónoma de Puebla.

Prezza, M., Amici, M., Roberti, T. y Tedeschi, G. (2001). Sense of community referred to the whole town: Its relations with neighboring, loneliness, life satisfaction, and area of residence. Journal of Community Psychology, 29(1), 29-52.

Reina, M., Vílchez, J., Ceballos, M. y López, J. (2017). Análisis de un proyecto de huerto escolar en secundaria a partir de las percepciones de los estudiantes. Enseñanza de las ciencias: revista de investigación y experiencias didácticas (pp. 1491-1496).

Richard, E. y Contreras, D. (2013). Reflexiones en torno a las reservas naturales urbanas como espacio de diálogo de saberes en la construcción de un ciudadano urbano crítico, responsable y comprometido con la problemática ambiental, la biofilia y la cultura de la contemplación para el buen vivir en Bolivia y Latinoamérica. Revista de Didáctica Ambiental, 9(13), 1-30.

Richter, F. y Cuenca, J. (2018). Huertos de ocio y vida comunitaria. La agricultura urbana como experiencia de participación ciudadana. En A. Madariaga y A. Ponce de León (coords.), Ocio y participación social en entornos comunitarios (pp. 189-212). Universidad de La Rioja.

Rodríguez, G., Gil, J. y García, E. (1996). Metodología de la investigación cualitativa. Ediciones Aljibe.

Romá-Ferri, T., Arroyo, H., y Aguiló, A. (coord.) (2017). Actas del Congreso Iberoamericano de Universidades Promotoras de la Salud: Promoción de la Salud y Universidad. Construyendo Entornos Sociales y Educativos Saludables. Universitat d'Alacant.

Santa-Cruz, A. (2015). El huerto urbano como ejemplo de transición hacia un modelo "ocialista": Símbolo y práctica de ocio creativo y sostenible en el centro de la ciudad. En A. Makua y I. Rubio (coords.). El legado de la crisis: respuestas desde el ámbito del ocio ( $p$. 55-72). Servicio de Publicaciones Argitalpen Zerbitzua. 
Sarandón, S. y Flores, C. (2014). Agroecología: bases teóricas para el diseño y manejo de agroecosistemas sustentables. La Plata, Argentina: Editorial de la Universidad Nacional de La Plata (EDULP).

Sen, A. (1987). Commodities and capabilities. Oxford UP.

Shen, L. Y., Ochoa, J. J., Shah, M. N. y Zhang, X. (2011). The application of urban sustainability indicators. A comparison between various practices. Habitat international, 35(1), 17-29.

Specht, K., Siebert, R., Hartmann, I., Freisinger, U. B., Sawicka, M., Werner, A. y Dierich, A. (2014). Urban agriculture of the future: an overview of sustainability aspects of food production in and on buildings. Agriculture and human values, 31(1), 33-51.

Suquilanda, M. (1996). Agricultura orgánica, alternativa tecnológica del futuro. Editorial UPS.

Tranfield, D., Denyer, D. y Smart, P. (2003). Towards a methodology for developing evidence-informed management knowledge by means of systematic review. British Journal of Management 14(3), 207-222.

Trescastro-López, E. y Trescastro-López, S. (2013). La educación en alimentación y nutrición en el medio escolar: el ejemplo del Programa EDALNU. Revista Española de Nutrición Humana y Dietética, 17(2), 84-90.

Vílchez, J. y Escobar, T. (2014). Uso de laboratorio, huerto escolar y visitas a centros de naturaleza en Primaria: Percepción de los futuros maestros durante sus prácticas docentes. Revista Electrónica de Enseñanza de las Ciencias, 13(2), 222-241.

Völker, B., Flap, H. y Lindenberg, S. (2007). When are neighbourhoods communities? Community in Dutch neighbourhoods. European Sociological Review, 23(1), 99-114.

Wen Li, W., Hodgetts, D. y Ho, E. (2010). Gardens, transitions and identity reconstruction among older Chinese immigrants to New Zealand. Journal of Health Psychology, 15(5), 786-796. 